\title{
ON THE HASSE PRINCIPLE FOR QUADRATIC FORMS
}

\author{
J. S. HSIA ${ }^{1}$
}

ABSTRACT. Examples are given for rational function fields that do not satisfy the strong Hasse principle for quadratic forms.

One of the most celebrated results in the theory of quadratic forms is the Hasse-Minkowski Theorem. It asserts that two quadratic forms over an algebraic number field are equivalent if and only if they are locally (everywhere) equivalent. This result is generally brought forth as a consequence of the following stronger version of the so-called "local-global principle": a quadratic form over a number field is isotropic (i.e. represents zero nontrivially) if and only if it is isotropic everywhere locally; see [BS], [OM]. What is, however, sometimes not recognized is that there are fields which satisfy the weak-but not the strong-Hasse principle. In this note we shall furnish some examples of rational function fields that do not satisfy the strong Hasse principle, and also make a few remarks about the two versions of the Hasse principle.

Let $F$ be a field of characteristic different from 2, and $S$ a set of inequivalent valuations on $F$. The members of $S$ shall be denoted by the p's. We say a quadratic form $\phi$ over $F$ satisfies the Strong Hasse Principle (SHP) w.r.t. $S$ if $\phi_{\mathfrak{p}}$ is locally isotropic at each $\mathfrak{p} \in S$ implies $\phi$ is isotropic. (As usual, locally here means with respect to the completion $F_{\mathfrak{p}}$ of $F$ at p.) The pair $(F, S)$ satisfies SHP if every quadratic form over $F$ satisfies SHP w.r.t. $S$. Two quadratic forms over $F$ satisfy the Weak Hasse Principle $(W H P)$ w.r.t. $S$ if local equivalence between them at each $\mathfrak{p} \in S$ implies global equivalence. Similarly, the pair $(F, S)$ satisfies WHP if every two quadratic forms over $F$ satisfy WHP w.r.t. $S$. It is immediate that SHP always implies WHP. For low enough dimensions, the converse is also valid; namely:

(i) If WHP holds for forms with dimension $\leqq 3$ w.r.t. $S$, then so does $S H P$.

Received by the editors November 3, 1972.

AMS (MOS) subject classifications (1970). Primary 10C05, 15A63; Secondary 10J05, $14 \mathrm{H} 05$.

Key words and phrases. Strong Hasse principle, weak Hasse principle, quadratic forms, sums of squares, rational function fields, isotropy, reduced height.

${ }^{1}$ This work was partially supported by the National Science Foundation Grant GP-23656.

(c) American Mathematical Society 1973 
Proof. One sees readily that when $\operatorname{dim} \phi=2$, then $\phi \cong(1,-1)$. And when $\operatorname{dim} \phi=3$, then $\phi \cong(1,-1) \perp(-\operatorname{det} \phi)$.

(ii) If $\phi$ is a quaternary form over $F$ with $\operatorname{det} \phi=1$, and WHP holds for 4-dimensional forms w.r.t. $S$, then $\phi$ satisfies $S H P$ w.r.t. S. More generally, if for every quadratic extension $K / F$ we assume WHP holds for 4-dimensional forms over $K$ w.r.t. $T$ where $T$ denotes the set of all valuations on $K$ which extend from $S$ on $F$, then every quaternary form over $F$ satisfies $S H P$ w.r.t. $S$.

Proof. If $\phi$ is quaternary and $\operatorname{det} \phi=1$, then $\phi \cong a(1, b) \otimes(1, c)$. Since it is locally isotropic, we must have then $\phi \cong(1,1,-1,-1)$. In the other cases, one considers the quadratic extension $K=F\left((\operatorname{det} \phi)^{1 / 2}\right)$.

(iii) SHP is satisfied by the pair $(F, S)$ where (a) $F$ is a global field (i.e. an algebraic number field or a function field in one variable over a Galois field) and $S$ the set of all spots on $F$ (see [OM]), (b) $F=\operatorname{Reals}(X)$ and $S$ either the set of all spots or else the set of all "finite" spots on $F$ that are trivial on the constants (see $[\mathbf{K}]$ ).

(iv) THEOREM OF HARDER-MILNOR. For any rational function field $F=k(X), \operatorname{Char}(k) \neq 2$, and $S$ the set of all spots that are trivial on $k$, the pair $(F, S)$ satisfies WHP. See ([Kn], [M]).

(v) ReMARK. The usual proof of the SHP for global fields (see [OM]) uses results from global class field theory (at least for quadratic extensions) to handle the cases of dimension $\leqq 3$, and then the higher dimensional cases follow from simple arithmetical and algebraic manipulations. We shall see below that there are rational function fields that do not satisfy SHP. Since they always satisfy WHP (whose proof requires no class field theoretic machinery) the comment made in (i) above is somewhat interesting. It is also worth mentioning here that whenever $(F, S)$ satisfies WHP, a result like the so-called "Global Square Theorem" (see [OM]) in a milder version is valid. Milder here means in the context that an element of $F$ must be, of necessity, a square at every $\mathfrak{p} \in S$ as opposed to merely at almost.all $\mathfrak{p} \in S$.

(vi) Theorem of Cassels-Ellison-Pfister. Let $k=\operatorname{Reals}(X)$. Then, the rational function field $F=k(Y)$ does not satisfy SHP.

Proof. (See [CEP].) The Motzkin polynomial

$$
f(X, Y)=1+X^{2}\left(X^{2}-3\right) Y^{2}+X^{2} Y^{4}
$$

is locally a sum of three squares but not globally (although it is a sum of four squares).

REMARK. The full proof of the theorem is quite nontrivial and uses the theory of elliptic curves. We shall, however, give examples below that 
require the more routine knowledge of quadratic form theory. We mention also here that WHP already fails for quadratic extensions of rational function fields. Indeed, if WHP were valid for every quadratic extension $K$ of $F=\boldsymbol{R}(X, Y)$, then it follows from (ii) that the quaternary form $\phi=(1,1,1,-f(X, Y))-f(X, Y)$ the Motzkin polynomial-satisfies SHP, contradicting (vi).

(vii) ExAmpLe. Let $k$ be a formally real algebraic number field. The reduced height $m(k)$ of $k$ (see $[\mathbf{H J}])$ is the least positive integer such that every totally positive element in $k$ is a sum of already $m(k)$ number of squares in $k$. In [HJ] we showed that $m(k)=3$ or 4 . It is 4 if and only if there is a dyadic prime $\mathfrak{q}$ (i.e. $q \mid 2)$ on $k$ such that the local degree $\left[k_{\mathrm{q}}: \boldsymbol{Q}_{2}\right]$ is odd (e.g., when $k$ has $[k: Q]$ an odd integer). Now, consider the case where $m(k)=3$ (e.g., $k=\boldsymbol{Q}(\sqrt{ } d), d>0$ and square-free and $\not \equiv 1(\bmod 8))$. Choose an element $b \in k$ such that $b$ is a sum of exactly (i.e. no fewer than) 3 squares in $k$. By a well-known result of Cassels, the polynomial $g(X)=$ $X^{2}+b$ cannot be represented as a sum of 3 squares in $k(X)$. Yet, by the local characterization of $k$ with $m(k)=3, g(X)$ is a sum of 3 squares everywhere locally. Hence, SHP is not satisfied by $F=k(X)$. By a similar argument as above, the quadratic extension field $K=F\left((-g(X))^{1 / 2}\right)$ does not satisfy WHP. Note: $K / F$ is a function field of genus zero.

\section{REFERENCES}

[BS] Z. I. Borevič and I. R. Safarevič, Number theory, "Nauka", Moscow, 1964; English transl., Pure and Appl. Math., vol. 20, Academic Press, New York, 1966. MR 30 \#1080; MR 33 \#4001.

[CEP] J. W. S. Cassels, W. J. Ellison and A. Pfister, On sums of squares and on elliptic curves over function fields, J. Number Theory 3 (1971), 125-149.

[HJ] J. S. Hsia and R. P. Johnson, On the representation in sums of squares for definite functions in one variable over an algebraic number field, Amer. J. Math. (to appear).

[Kn] M. Knebusch, Grothendieck und Wittringe von nichtausgearteten symmetrischen Bilinearformen, S.-B. Heidelberg, Akad. Wiss. 1969/70, 93-157. MR 42 \#6001.

[K] J. T. Knight, Quadratic forms over $R(t)$, Proc. Cambridge Philos. Soc. 62 (1966), 197-205.

[M] J. Milnor, Algebraic K-theory and quadratic forms, Invent. Math. 9 (1969/70), 318-344. MR 41 \#5465.

[OM] O. T. O'Meara, Introduction to quadratic forms, Die Grundlehren der math. Wissenschaften, Band 117, Academic Press, New York; Springer-Verlag, Berlin, 1963. MR 27 \#2485.

Department of Mathematics, Ohio State University, Columbus, Ohio 43210 